Irish Math. Soc. Bulletin

Number 75, Summer 2015, 5-6

ISSN 0791-5578

\title{
FLAT SURFACES OF FINITE TYPE IN THE 3-SPHERE
}

\author{
ALAN MCCARTHY
}

This is an abstract of the $\mathrm{PhD}$ thesis Flat surfaces of Finite Type in the 3-Sphere written by Alan McCarthy under the supervision of Martin Kilian at the School of Mathematical Sciences, University College Cork, and submitted in May 2014.

The local theory of flat surfaces in $\mathbb{S}^{3}$ through the use of asymptotic curves in $\mathbb{S}^{3}$ was already known by Bianchi [1], however the problem of classifying flat tori was first posed by Yau [4] in 1974. Kitagawa 2 provided a classification by using asymptotic lifts of 'admissible pairs' of closed curves on $\mathbb{S}^{2}$. Flat tori were also classified by Weiner [3] in terms of their Gauss maps.

My thesis is concerned with finite gap flat surfaces. These are surfaces whose generating curves on $\mathbb{S}^{2}$ have finite gap geodesic curvatures, which means that eventually all flows of the mKdV hierarchy are finite linear combinations of preceeding ones.

We provide a summary of finite gap curves in terms of Lax pairs, Killing fields, their spectral curves and provide conditions that ensure that the curve remains closed and spherical. We also provide a discussion of the isoperiodic deformations and monodromy associated to the frame of the curves.

As an application we show that given an admissible pair of curves $\gamma_{1}, \gamma_{2}$ with geodesic curvatures $k_{1}, k_{2} \in L^{2}\left(\mathbb{S}^{1}, \mathbb{R}\right)$, there exists a pair of finite gap curvature functions that generate curves on $\mathbb{S}^{2}$ that are admissible and that these finite gap curvatures are also dense in the Sobolev norm.

2010 Mathematics Subject Classification. 51H25, 35P30,58A02,53C02.

Key words and phrases. differential geometry, integrable systems, flat, tori, finite type, finite gap.

Received on 23-1-2015.

Support from University College Cork through a part time lecturship is gratefully acknowledged. 


\section{REFERENCES}

[1] L. Bianchi. Sulle superficie a curvatura nulla in geometria ellittica. Ann. Mat. Pura Appl., 24(1):9-19, 1896.

[2] Y. Kitagawa. Periodicity of the asymptotic curves on flat tori in $S^{3}$. J. Math. Soc. Japan, 40(3):457-476, 1988.

[3] J. L. Weiner. Flat tori in $S^{3}$ and their Gauss maps. Proc. London Math. Soc. (3), 62(1):54-76, 1991.

[4] S. T. Yau. Submanifolds with constant mean curvature. I, II. Amer. J. Math., 96:346-366; ibid. 97 (1975), 76-100, 1974.

(from August 2014) School of Mathematics And Statistics, UniverSity of New South Wales, Sydney

E-mail address, A. McCarthy: a.mccarthy@unsw.edu.au 